\title{
The role of direction information in the perception of geometric optic flow components
}

\author{
BART DE BRUYN and GUY A. ORBAN \\ Catholic University of Louvain, Louvain, Belgium
}

\begin{abstract}
Theoretically, optic flow, an important source of information for the perception of locomotion and three-dimensional structure of the environment, is described in terms of divergence, curl, and shear components. We measured how the detection of the type of flow field depends on directional information. We manipulated the local directions by rotating them through an angle $x$ relative to the original direction (i.e., the direction of motion at that locus in an unaltered flow field). The results of the first experiments showed that divergence, curl, and shear can be detected even if the directional range of the individual motion vectors is as broad as $180^{\circ}$. Subsequent experiments revealed that the detection of the geometric components of the optic flow field is merely based on the integration of a few ( $10 \%$ of vectors) local directions correctly (within $10^{\circ}$ of original direction) specifying the type of flow field. Other directions are irrelevant to this process. This is actually what one would expect if the optic flow is analyzed by special purpose mechanisms that detect and process the geometric components on the basis of the integration of motion information. The results indicate that as far as they integrate motion information, detectors for divergence, curl, and shear operate in a similar manner. Implications of the results for modeling such mechanisms are discussed.
\end{abstract}

Changes in the optic array, a term coined by Gibson (1950) to denote the bundle of labeled visual directions (Koenderink, 1986), are due to egomotion or physical motions of external objects. The spatiotemporal change of the optic array or optic flow is therefore considered to be a rich source of information about locomotion and the three-dimensional layout of the environment. Although the theoretical existence of this information in itself does not guarantee that it will be used, several experimental studies have shown that optic flow is an effective stimulus for the visual system (for references, see Koenderink, 1986; Pradzny, 1983; Warren, Morris, \& Kalish, 1988). Formal analysis, based on differential derivatives, has described the optic flow field in terms of divergence, curl, and shear components (Koenderink, 1985, 1986; Koenderink \& van Doorn, 1976, 1978; Longuet-Higgins \& Pradzny, 1985). Psychophysical and physiological evidence for mechanisms sensitive to divergence and curl is provided in the work of Regan and Beverley (1978, $1979,1984)$ and Saito et al. (1986). As pointed out by Koenderink (1986), the extraction of the geometric components of the optic flow can take place in different ways. Most often stressed is the use of local motion measurements. Mechanisms for detecting and processing the geometric components of the optic flow can be constructed by connecting several local motion detectors detecting

The technical help of P. Kayenbergh, G. Meulemans, G. Vanparrijs, and $Y$. Celis is gratefully acknowledged. B. De Bruyn was supported as a research assistant by the National Fund for Scientific Research. Correspondence may be addressed to Bart De Bruyn, Laboratorium voor Neuro -en Psychofysiologie, Katholieke Universiteit Leuven, Campus Gasthuisberg, Herestraat, B-3000 Leuven, Belgium. different directions at different places in the visual field. In the present experiments, we have measured the visibility of the geometric components of the optic flow field, assuming that the processing of these components is based on local motion information.

A geometric component of the optic flow field can be described by a vector function that specifies a magnitude (speed) and a direction at every point in a certain region of space. As a stimulus, we used a set of randomly placed dots, which were carried along the flow. The flow is then specified by a limited number of vectors and not by a continuous field (Koenderink \& van Doorn, 1987a). Four types of flow fields were tested (see Figure 1). They include the three geometric components of optic flowdivergence, curl, and shear-and, as a fourth one, a simple unidirectional translation. Two superimposed sequential images of our kinematic displays would be similar to the patterns described by Glass (1969), Glass and Perez (1973), Glass and Switkes (1976), and Prazdny (1986). A flow field describing a pure geometric component on the basis of motion has two sources of information. The first is local speed and its distribution over an extended area; the second is local direction and its distribution.

A completely random distribution of local speeds destroys the spatial coherence of the dots and leads to perception of transparency and different depth planes, whereas the speed gradient provides information about three-dimensional shape (De Bruyn \& Orban, in press). However, in both cases, the geometric optic flow component can still be detected. In contrast with local speed and its gradient, the local directions of the motion vectors are essential for specifying the type of geometric component. A complete randomization of local directions (i.e., 


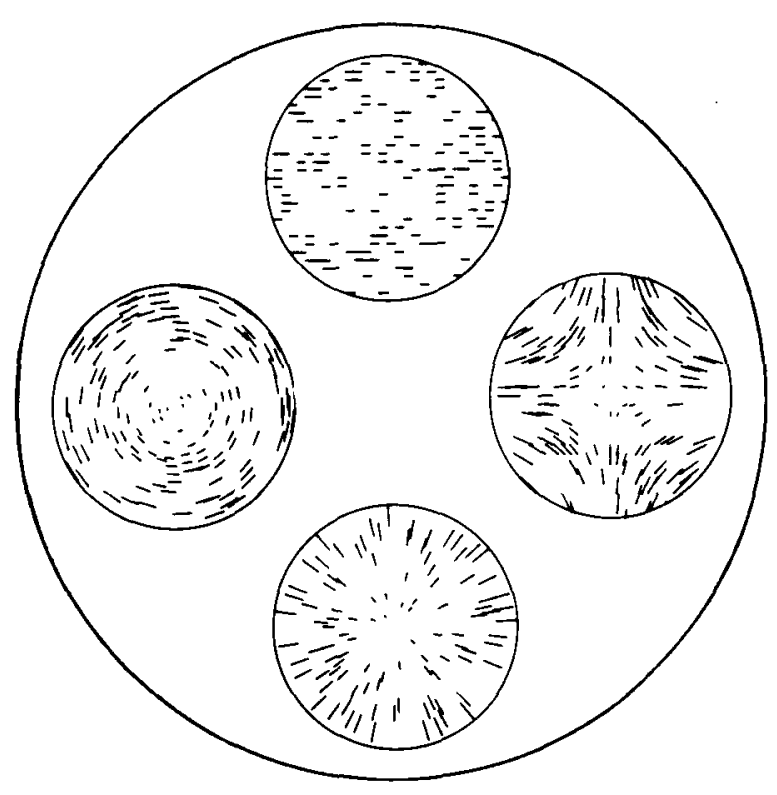

Figure 1. The flow fields shown resulted from superimposing the original dot pattern with a number of frames of transformed dot patterns. Four transformations were used: at the top a translation, and, clockwise from the top, a shearing, a divergence, and a rotation. Mlustrated is the display containing the sample stimulus. Four "ideal" types of the flow fields are presented within a circular aperture equal in size to the test flow field. The density of the dots in the ideal types is higher than in the test flow fields. These ideal types could be easily detected and discriminated by the subject. After a test flow field was presented, the subject had to match the flow field just seen with one of the ideal types present in the sample.

a selection of local motion vectors from a directional range of $360^{\circ}$ ), destroys a coherent global percept. This is also shown in the work of Glass and Switkes (1976) and Williams and Sekuler (1984). That local directions specify the type of geometric component can be easily understood if one considers a case in which all local directions are rotated through a fixed angle relative to their original, unaltered direction. An overall local rotation of $90^{\circ}$, for instance, changes an expanding flow field into a rotating one. In a similar manner, components of shear can be transformed into each other. An overall local rotation of $45^{\circ}$ will yield similar spiral-like patterns for expansion and rotation. Our question is how clearly each type of flow field can be identified. As argued above, the way to approach this question is to compare the effect of variations in local directions on the detection of the geometric components of optic flow.

\section{METHOD}

\section{Equipment}

Stimuli were generated on an Atari SM124 display driven by an Atari MegaST4 microcomputer. Sequential patterns were presented at a frame rate of $35 \mathrm{~Hz}$.

\section{Subjects}

One of the authors (B.D.B.) and K.L., who was unaware of the purpose of the experiment, served as subjects. Both subjects had normal or corrected vision.

\section{Stimuli}

The stimuli were computer-generated white dots of fixed size (2 pixels in diameter) plotted on a black background. For the first frame, dots were generated with a probability of 0.2 and positioned in a two-dimensional matrix, each cell of which was $10 \times 10$ pixels in size. In order to avoid effects of spatial alignment, a random value, selected between -5 and 5 pixels, was added independently to the initial $x$ and $y$ positions. The random-dot patterns were only visible in a circular aperture with a diameter of $10^{\circ}$. On the average, 180 dots were plotted. Dot positions in sequential frames were changed by two-dimensional transformation functions. The following transformations were used: a translation in the horizontal axis, $x^{\prime}=x+D x, y^{\prime}=y$; a rotation or curl, $x^{\prime}=x \cos \phi-y \sin \phi$, $y^{\prime}=x \sin \phi+y \cos \phi$; a divergence, $x^{\prime}=x S x, y^{\prime}=y S y$; and a shearing, same transformation as the expansion but the scaling factors $(S x, S y)$ with opposite signs. In the formulas, $x^{\prime}$ and $y^{\prime}$ refer to the transformed $x$ and $y$ coordinates of a dot. It should be noted that for the divergence, the scaling factors $S x$ and $S y$ are equal to one another and greater than one. This transformation results in a uniform, homogeneous stretch that does not change the proportions and is equal to a zooming or magnification. At least for a small aperture and for short presentation times, this transformation represents the flow field for an observer moving toward a planar surface.

A series of sequential frames will be referred to here as a movie. The direction of the transformation (e.g., expansion and contraction) was varied by playing a movie forward or backward. The speed of the dots of the flow fields describing the geometric components ranged from $0 \% \mathrm{sec}$ at the center to $8 \% \mathrm{sec}$ at the periphery. A stationary dot at the center was left out. All the dots of the translatory flow field moved at a speed equal to the average of the speeds theoretically present in flow fields describing the geometric components. Each movie consisted of five frames. The frames were sequentially presented at a refresh rate of $35 \mathrm{~Hz}$, resulting in a stimulus duration of $115 \mathrm{msec}$. A new random-dot pattern and its transformed versions were calculated for every trial. The type of the flow field as well as the direction of change (e.g., expansion vs. contraction) was presented in a randomly interleaved manner. Only one component of shear was used (contraction in the horizontal axis accompanied by an expansion in the perpendicular axis; see Koenderink \& van Doorn, 1976, 1978). There is no a priori reason for assuming a difference in visibility between the flow fields corresponding to the two components of shear. We varied the local directions by changing the orientation of the motion vectors present in the flow field. Without changing the local speed, local motion vectors were rotated about the original dot position through an angle $x$ relative to the original orientation (Figure 2). In contrast with the shuffle technique used by Andersen and Siegel (in press) and Spitz, Stiles-Davis, and Siegel (1988), we changed only the directional information.

\section{Procedure}

Detection of the type of flow field was measured in a matchingto-sample task. This method was chosen in order to avoid explicit verbal labeling in the instructions to the subject. A sample stimulus appeared directly after the presentation of the flow field. This sample stimulus contained "ideal" types of the four types of flow fields (Figure 1). The sample movie was displayed in a sequence of the four flow fields undergoing change in one direction (rightward translation, expansion, clockwise rotation, shear with expansion in the horizontal axis) followed by the four fields undergoing 

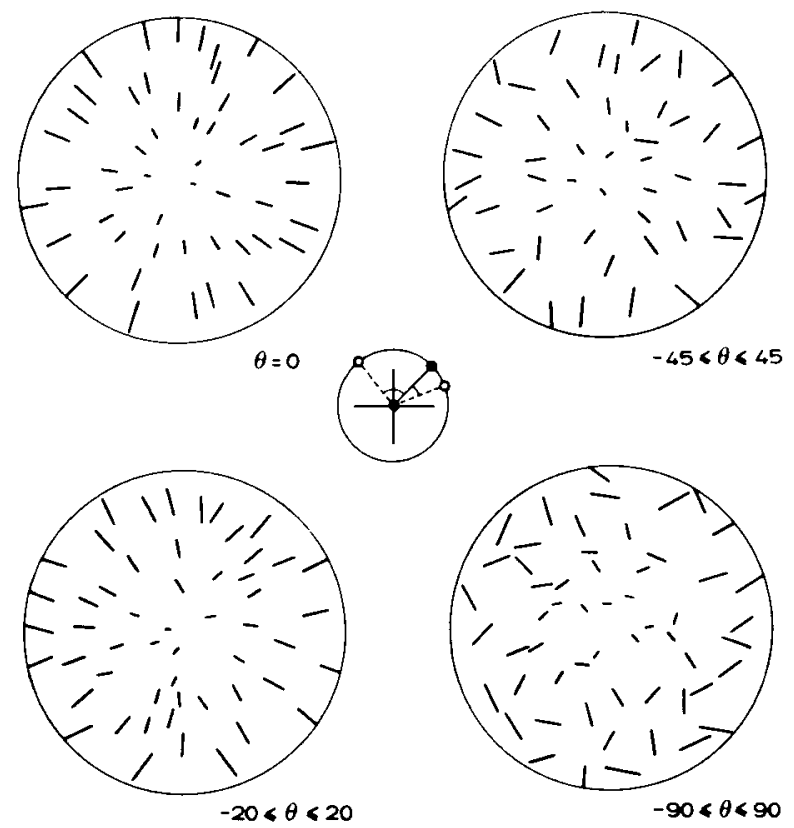

Figure 2. The expansion pattern shown at the upper left results from superimposing the original dot pattern with four frames of transformed dot patterns. Each line indicates a motion vector, the orientation of the line specifies the direction, and the length specifies the speed. All vectors in this expansion pattern are in the original, unaltered direction-radiating outward. In the other three patterns, local motion vectors are rotated relative to their original orientations. This is illustrated at the center of the figure. The vector from the center toward the filled dot illustrates the original direction. The vectors defined by the center and the open dots illustrate two possible rotations, through angles of $20^{\circ}$ and $80^{\circ}$, relative to the original orientation. If a relative rotation is assigned to a given local motion vector, the dot will move according to this new vector during the whole movie. The range from which the angle of rotation is randomly selected is indicated for each pattern.

change in the opposite direction (leftward translation, contraction, counter-clockwise rotation, and shear with contraction in the horizontal axis). This sample sequence was repeated until the observer responded. The observer had to match the flow field just presented with one of the four ideal types of the sample. The psychophysical method used to measure visibility was a transformed up-down method (TUDM) (Levitt, 1971; Wetherill \& Levitt, 1965). In the first two experiments, the experimental variable was decreased by $20 \%$ after three successive correct responses and was increased by $20 \%$ after one incorrect response. In Experiment 3, tracking the number of dots moving in an unaltered direction, we used a fixed rather than a proportional step size. The staircase algorithm tracks a 79\% correct response level. The TUDM was stopped after 6 reversal points. The geometric mean of the last 3 reversal points was taken as the threshold. The TUDMs for the four types of flow fields were running at the same time. In order to prevent the observer from following the tracking and thereby using the differences between the four TUDMs as cues for detectability, dummy trials were included. For a dummy trial, experimental parameters were randomly selected. Control experiments showed that an observer could not follow the staircase procedure if $30 \%$ of all presentations were dummies. Responses on these trials were discarded. Every measurement was repeated at least three times. The observer, supported by a chinrest, viewed the display monocularly from a dis- tance of $57 \mathrm{~cm}$. Two push-buttons connected to the computer signaled the observer's response. At the beginning of each trial, a fixation point was presented. A tone signaled the start of the movement. No feedback was given.

\section{RESULTS}

\section{Experiment 1: Maximum Range of Local Directions}

Starting with the unaltered flow fields, we tracked the maximum directional range from which the direction of local motion vectors can be selected without limiting the detection of the type of flow field. The direction of a local motion vector was changed by rotating the vector about an angle relative to the original direction (Figure 2). The range from which this rotation angle was randomly selected was subjected to the TUDM and therefore changed according to the responses of the subject. Three consecutive correct responses increased this range by $20 \%$. An incorrect response decreased the range by $20 \%$. Pretesting showed that the flow fields could be detected accurately when the angle was chosen from an interval centrally bisected by the original direction, of $90^{\circ}$. The TUDMs were therefore started with flow fields in which the local rotation angles were randomly selected between $-45^{\circ}$ and $+45^{\circ}$. The results for both subjects are shown in Figure 3. Clearly, there was little difference between repeated measurements or between subjects. The average ranges of directions and standard deviations ( 2 subjects, six measurements) were: $193^{\circ} \pm 5.1^{\circ}$ for translation, $194^{\circ} \pm 6.8^{\circ}$ for curl, $186^{\circ} \pm 4.5^{\circ}$ for divergence, and $188^{\circ} \pm 4.8^{\circ}$ for shearing. The average values represent the range of directions, in degrees, which was centrally bisected by the original direction. Irrespective of the type of flow field, detection of the type of flow field is possible if the local directions are selected from a directional range of up to $190^{\circ}$. Theoretically, the maximum range should be $180^{\circ}$. Indeed, angles outside this range would merely provide information for the reversed direction. The step-size criterion used, which in our experiment was $20 \%$, can account for minor deviations from the expected range of $180^{\circ}$.

One interpretation of the results of Experiment 1 is that the visual system can integrate different local direction measurements into the perception of a coherent motion, by computing, for example, the vectorial mean of motion vectors. An alternate interpretation is that when local directions are taken randomly from a uniform distribution of $180^{\circ}$, unaltered directions that are also present suffice to determine the type of flow field. We therefore wanted to test whether or not the detection of the geometric components of the optic flow field is merely based on the integration of a few local, unaltered directions, other directions being irrelevant. This is actually what one would expect if the optic flow is analyzed by special purpose mechanisms that detect and process the geometric components of the optic flow. 

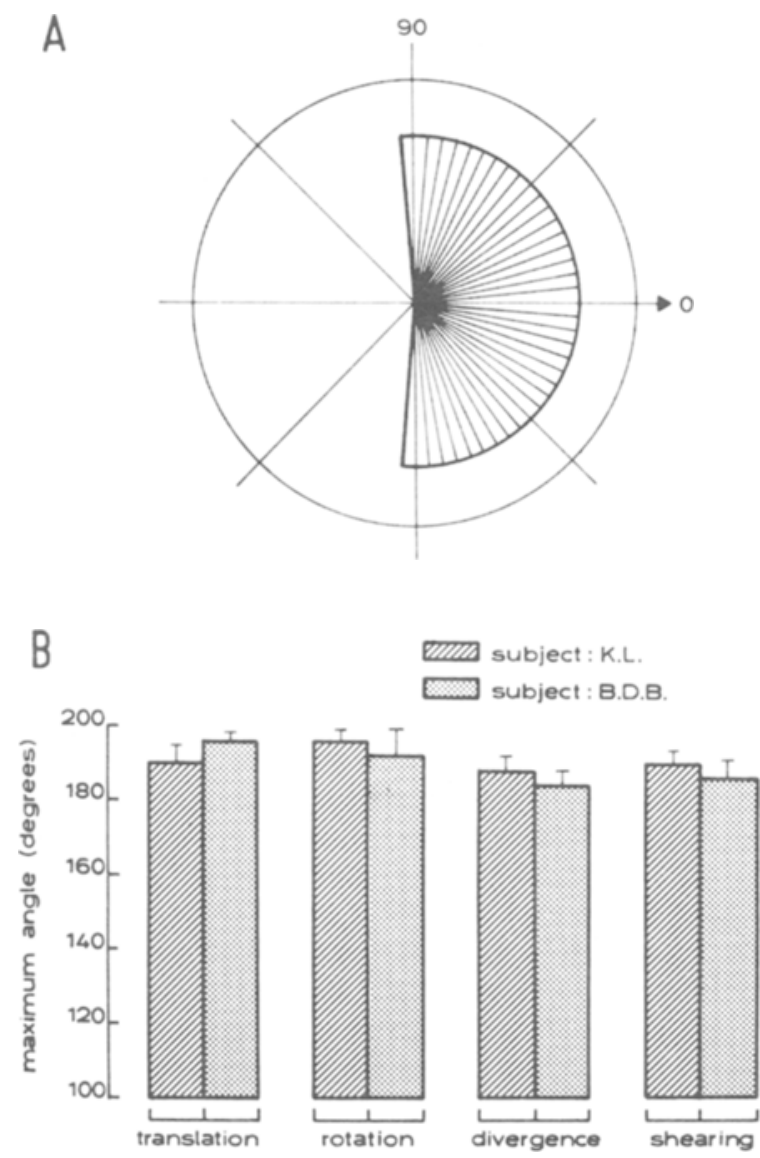

Figure 3. The maximum range, expressed as an angle centrally bisected by the original direction, from which local rotation angles can be randomly selected without limiting the detection of the type of flow field. (A) Graphical illustration of the maximum range: the value shown $\left(190^{\circ}\right)$ corresponds to the average for all types of flow fields. (B) Means and standard deviations $(n=3)$ of the maximum ranges for both subjects. The arrow in (A) indicates the unaltered direction of the local motion vector.

\section{Experiment 2: In Search of Noise Directions}

Before we could track the number of dots moving in unaltered directions necessary for identifying the flow fields, we needed to determine the noise directions. The question is, which angles have to be excluded from a flow field in which local rotation angles are selected from a uniform distribution of $180^{\circ}$ so that the detection of the type of flow field is no longer possible? The angles remaining will then represent the noise directions. We started the testing with flow fields in which local rotation angles were randomly drawn from the interval $-90^{\circ}$ to $-5^{\circ}$ and $5^{\circ}$ to $90^{\circ}$. No angles were taken out of the range between $-5^{\circ}$ and $5^{\circ}$, which was centrally bisected by the original local direction. The range from which no angles were selected was the experimental variable. The results for both subjects are shown in Figure 4. As in Experiment 1 , there was little difference between subjects or repeated measurements. The average results and standard deviations ( 2 subjects, six measurements) were: $22^{\circ} \pm 3.4^{\circ}$ for translation, $18^{\circ} \pm 3.3^{\circ}$ for curl, $20^{\circ} \pm 3.8^{\circ}$ for divergence, and $18^{\circ} \pm 3.1^{\circ}$ for shearing. The average values represent the ranges of directions in degrees, centrally bisected by the original direction, that could be excluded without limiting the detection of the type of flow field. This exclusion range of $20^{\circ}$ was similar for the four types of flow fields tested.

\section{Experiment 3: Number of Dots Moving in the Original Direction}

Since we now know the noise directions, we can measure the number of dots moving in the original direction necessary for the detection of the flow fields. The TUDMs were started with flow fields in which all local motion vectors were rotated by an angle randomly selected from a uniform distribution ranging from $-90^{\circ}$ to $-15^{\circ}$ and $15^{\circ}$ to $90^{\circ}$ with respect to the original direction. Hence, initially, all dots were "noise dots." If the subject could
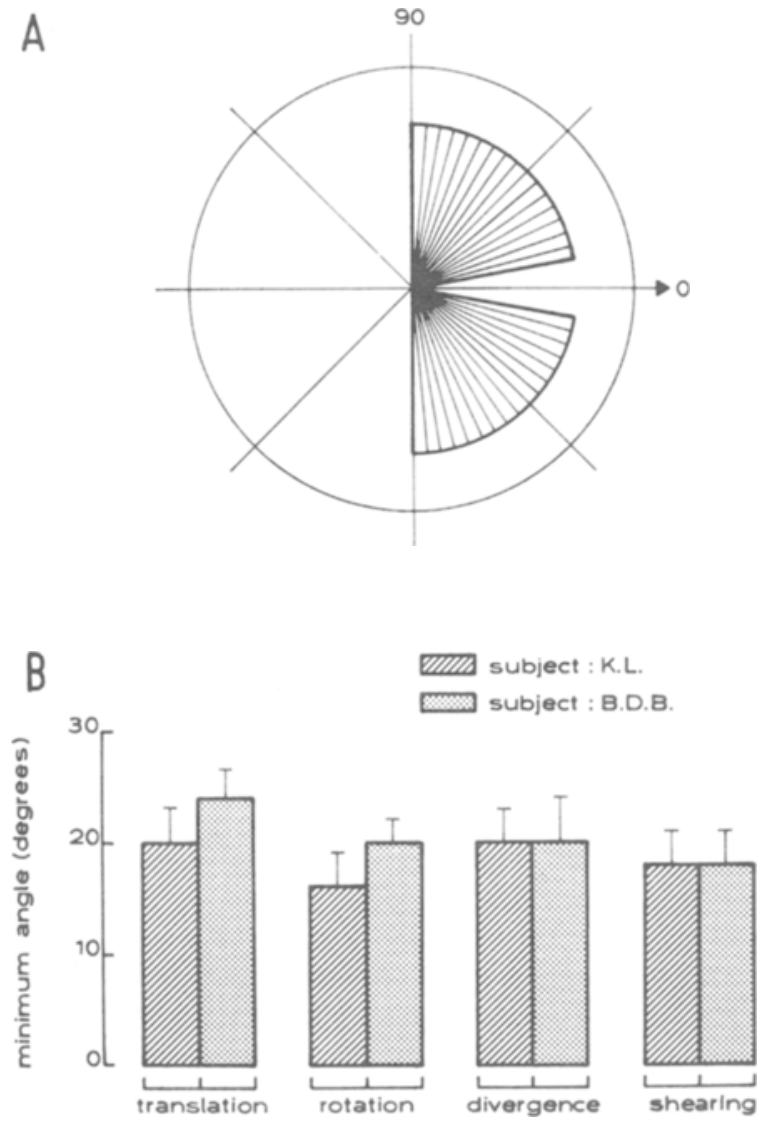

Figure 4. The minimum range of local rotation angles, expressed as an angle centrally bisected by the original direction of the local motion vector (indicated by the arrow), which can be excluded from a uniform distribution of $180^{\circ}$ without limiting the detection of the type of flow field. (A) Graphical illustration of the minimum range: the value shown $\left(20^{\circ}\right)$ corresponds to the average for all types of flow fields. (B) Means and standard deviations $(n=3)$ of the minimum ranges for both subjects. 

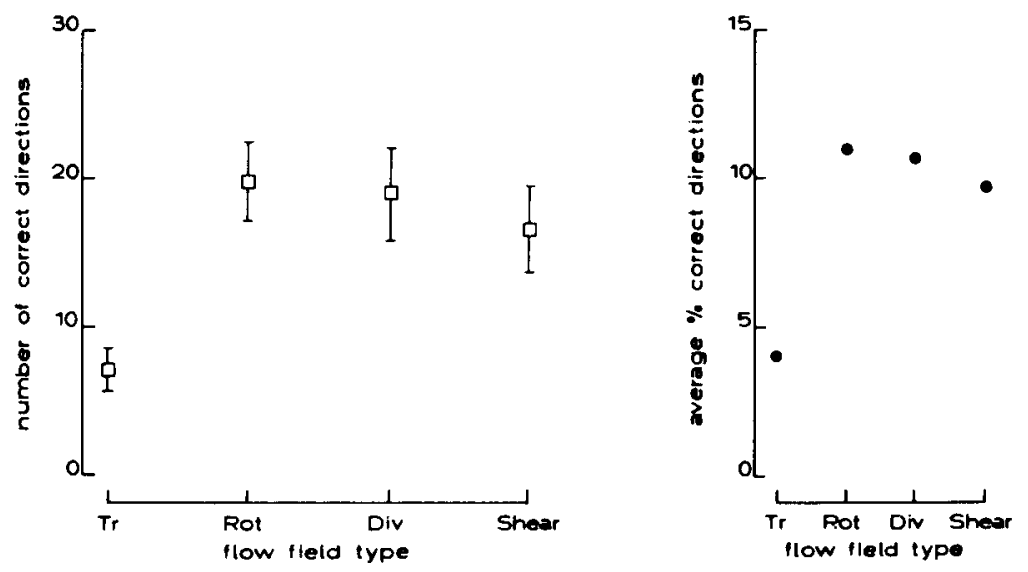

Figure 5. Numbers of dots that have to move in the original directions in a flow field in which all other local directions are rotated by an angle randomly selected from the range of $-90^{\circ}$ to $-15^{\circ}$ and $15^{\circ}$ to $90^{\circ}$ for the type of flow field to be detectable, plotted as a function of flow field type, translation (Tr), rotation or curl (Rot), divergence (Div), and shear. Vertical bars indicate standard deviations. In the right panel, the same results are expressed in average percentages. The results consist of averaged data for 2 subjects (six measurements).

not identify the flow field, two "original" dots-dots moving, for example, in unaltered directions radiating outward for an expansion-would be present in the next presentation of the same flow field. The density of the dots was not changed, since noise dots were replaced by the same number of original dots. The position of the new unaltered directions can be critical. Care was taken to make sure that the positions of correctly moving dots were not cluttered. We selected new positions in a circular manner, so that when two correctly moving dots were present, they were separated on the circle by about $180^{\circ}$. Four dots would be separated by about $90^{\circ}$, and so on. The radius of the circle or the distance of a correctly moving dot from the center was randomly selected. The number of unaltered directions was incremented by two on every error made by the observer and decremented by two dots if three consecutive correct responses were made. The average results as well as standard deviations are shown in Figure 5. Expressed in precentages, translation was detected when about $4 \%$ of the dots, in an otherwise noisy flow field, moved in the original direction. For the detection of the geometric components of the optic flow, this proportion was doubled: about $10 \%$ of the dots had to move in the unaltered direction. This result shows that for detection of the type of flow field, only a few unaltered local directions need to be present.

\section{DISCUSSION}

In the present experiments, we tested the detection of the type of flow field based on the integration of local direction measurements. Only a few unaltered directions in an otherwise noisy display must be present for one to detect a pure geometric component of the optic flow. The results for the translational motion in our matching-to- sample task can be compared with the results of Williams and Sekuler (1984). Their study showed that if the trajectories of individual dots are drawn from a range of $180^{\circ}$, the observer will perceive a global translation in the direction of the mean of the distribution. Furthermore, they showed that in order to generate this percept a number of dots moving in unaltered directions must be present. The range of directions that could be deleted from the center of a direction distribution of $180^{\circ}$ is about $15^{\circ}-20^{\circ}$ (interpolated from the results of Williams and Sekuler, for a threshold level of $79 \%$ correct direction detection). The present study confirms their results for translation. This confirmation serves to validate our matching-tosample procedure. In addition, we determined the percentage of dots moving in unaltered directions that must be present for the perception of global translation. Furthermore, we showed that the results for translation can be generalized to more complex motions such as the geometric components of optic flow, with the exception that the number of unaltered directions required is larger than the number necessary for the detection of translation.

We did not observe a difference between the amounts of integration of local direction measurement required to form the global percept of curl, divergence, and shear. A difference between the percentage of unaltered directions that must be present for the detection of translation and that required for the detection of the geometric components is not too suprising. For detection of a translation, less directional information is needed than for the detection of an optic flow component. In the extreme case, a translation can be identified from a display with only one moving dot. For differentiation between a translation and the geometric components divergence and shear, as well as between divergence and shear themselves, more local direction measurements are required. The rotation 
component is a special case. For long presentation times, it is possible to differentiate translation and rotation on the basis of one moving dot. For short presentation times, however, this is no longer possible, and one will need more, theoretically two, local direction measurements. This line of argument indicates that a difference in detection between the geometric components can possibly be found at longer presentation times.

With respect to the difference between the detection of translation and that of the optic flow components, it should be noted that the speed gradient could be a confounding factor. Whereas for the optic flow components local speed gradually increases from zero at the center toward the periphery, for the translation all the dots moved at the same speed. It is, however, unlikely that the difference in speed gradient was a confounding factor. The results of the second experiment showed that the directional range that can be deleted is equal for all tested flow fields. This indicates that the speed gradient did not contribute to the detection of translation, as compared with the other flow fields.

The present experiments do not directly test the existence of mechanisms that detect and analyze the geometric components of optic flow based on local motion measurements. These special purpose mechanisms, based on the integration of local directions, are described in the theoretical studies of Koenderink (1986), Koenderink and văn Doorn (1978), and Longuet-Higgins and Prazdny (1985), and they are supported by the psychophysical work of Regan (1986) and the physiological studies of Saito et al. (1986). Our results indicate that if such mechanisms for detecting and processing divergence, curl, and shear use motion information, they all operate in a similar way. That is, the amounts of directional information required are equal, as is the $20^{\circ}$ direction resolution. The findings indicate that such mechanisms must be able to integrate different motion vectors at different retinal positions, taking into account only relevant directions. That the few correctly moving dots can be randomly distributed in space indicates that the input for these mechanisms is not spatially limited. Furthermore, other results (De Bruyn $\&$ Orban, in press) indicate that such mechanisms should be able not only to compare direction information at various positions but also to integrate local speed measurements as a function of position. The detection and processing of the optic flow components therefore requires multilocal algorithms (Koenderink \& van Doorn, 1987b), in which direction as well as speed information is taken into account.

\section{REFERENCES}

Andersen, R. A., \& Siegel, R. M. (in press). Motion processing in primate cortex. In G. Edelman, W. Gall, \& W. Cowan (Eds.), Signal and sense: Local and global order in perceptual maps. New York: Wiley.
DE BRUYN, B., \& ORBAN, G. A. (in press). The importance of velocity gradients in the perception of 3D-rigidity. Perception.

GiBson, J. J. (1950). The perception of the visual world. Boston: Houghton Mifflin.

GLass, L. (1969). Moire effect from random dots. Nature, 223, 578-580.

Glass, L., \& Perez, R. (1973). Perception of random dot interference patterns. Nature, 246, 360-362.

GLASS, L., \& SWTTKES, E. (1976). Pattern recognition in humans: Correlations which cannot be perceived. Perception, 5, 67-72.

KoENDERINK, J. J. (1985). Space, form and optical deformations. In D. J. Ingle, M. Jeannerod, \& D. N. Lee (Eds.), Brain mechanisms and spatial vision (NATO ASI Series: Series D. Behavioural and Social Sciences, No. 21, pp. 30-58). Dordrecht: Martinus Nijhof.

KoENDERINK, J. J. (1986). Optic flow. Vision Research, 26, 161-170.

KoEnderink, J. J., \& VAN DOORN, A. J. (1976). Local structure of movement parallax of the plane. Journal of the Optical Society of America, 66, 717-723.

KoEnderink, J. J., \& VAN DOORN, A. J. (1978). How an ambulant observer can construct a model of the environment from the geometrical structure of the visual inflow. In G. Hauske \& E. Butenand (Eds.), Kybernetik (pp. 224-247). Munich: Oldenburg.

KoEnderink, J. J., \& VAN DOORN, A. J. (1987a). Facts on optic flow. Biological Cybernetics, 56, 247-254.

Koenderink, J. J., \& VAN Doorn, A. J. (1987b). Representation of local geometry in the visual system. Biological Cybernetics, 55, 367-375

LevirT, H. (1971). Transformed up-down methods in psychoacoustics. Journal of the Acoustical Society of America, 49, 467-477.

Longuet-Higgins, H. C., \& Prazdny, K. (1985). The interpretation of a moving retinal image. Proceedings of the Royal Society of London, 208B, 385-397.

Prazdny, K. (1983). On the information in optical flows. Computer Vision, Graphics \& Image Processing, 22, 239-259.

Prazdny, K. (1986). Some new phenomena in the perception of glass patterns. Biological Cybernetics, 53, 153-158.

REGAN, D. (1986). Visual processing of four kinds of relative motion. Vision Research, 26, 127-145.

REgAN, D., BeverLey, K. I. (1978). Looming detectors in the human visual pathway. Vision Research, 18, 415-421.

REgAN, D., \& BEVERLEY, K. I. (1979). Visually guided locomotion: Psychophysical evidence for a neural mechanism sensitive to flow patterns. Science, 205, 311-313.

Regan, D., \& Beverley, K. I. (1984). Psychophysics of visual flow patterns and motion in depth. In L. Spillman \& B. R. Wooten (Eds.), Sensory experience, adaptation and perception: Festschrift for Ivo Kohler (pp. 215-240). Hillsdale, NJ: Erlbaum.

Saito, H., Yukie, M., Tanaka, K., Hikosaka, K., Fukada, Y., \& IWAI, E. (1986). Integration of direction signals of image motion in the superior temporal sulcus of the macaque monkey. Joumal of Neuroscience, 6, 145-157.

Spitz, R. V., Stiles-Davis, J., \& Siegel, R. M. (1988). Infant perception of structural rotation and expansion from structure-from-motion displays. Society for Neuroscience Abstracts, 14, 1244.

Warren, W. H., Morris, M. W., Kalish, M. (1988). Perception of translational heading from optical flow. Journal of Experimental Psychology: Human Perception \& Performance, 14, 646-660.

WeTheriLl, G. B., \& LevitT, H. (1965). Sequential estimation of points on a psychometric function. British Joumal of Mathematical \& Statistical Psychology, 18, 1-10.

Williams, D. W., \& Sekuler, R. (1984). Coherent global motion percepts from stochastic local motions. Vision Research, 24, 55-62.

(Manuscript received July 24, 1989; revision accepted for publication November $7,1989$. 